(C) 1991 IEEE. Personal use of this material is permitted. However, permission to reprint/republish this material for advertising or promotional purposes or for creating new collective works for resale or redistribution to servers or lists, or to reuse any copyrighted component of this work in other works must be obtained from the IEEE.

\title{
Design Of A Multivariable RF Control System Using Gain-Shaping In The Frequency Domain*
}

\author{
Christopher D. Ziomek, Stephen P. Jachim, Eckard F. Natter \\ MS-H827, Los Alamos National Laboratory, Los Alamos, NM 87545
}

\section{Abstract}

Due to the time-varying nature of the radio-frequency (RF) accelerator, RF field amplitude and phase parameters must be precisely controlled in order to confine and accelerate the charged particle beam. Typically, a feedback control system regulates the $\mathrm{RF}$ field, rejects noise and disturbances, and maintains operational stability over changes in the electrical structure of the accelerator. This paper describes a multivariable control system that compensates the electrical structure of the accelerator by using gain-shaping in the frequency domain. The amplitude and phase quantities have been resolved into in-phase and quadrature (I\&Q) variables. These orthogonal variables have simple mathematical relationships, and can be analyzed using linear transfer function matrices. The transfer matrix theory has been applied to the design of the multivariable control system that regulates the RF field in-phase and quadrature components. Frequencydomain controllers compensate these two signals to provide desired frequency response characteristics. A control predistorter performs an inverse coupling function, so that the $I \& Q$ components are effectively decoupled by the accelerator. Furthermore, computer interface circuitry allows the adaptive optimization of the mathematical transfer functions of the compensators.

\section{INTRODUCTION}

The radio-frequency (RF) accelerator uses a time-varying electromagnetic field to excite charged particles to high kinetic energies. Resonant structures support electromagnetic oscillations having a travelling wave component with a phase velocity of approximately the same velocity as that of the accelerated particles. Precision RF instrumentation is required to control and monitor the amplitude and phase of the time-varying RF field within the accelerating cavity. A two-input, two-output control system is needed to accurately maintain both the amplitude and the phase at a desired operating point. Since coupling between the two control loops can severely degrade the system performance, the control system must include a decoupling network to negate any interaction between the two control variables. The Los Alamos National Laboratory (LANL) has designed a multivariable feedback control system that regulates the in-phase and quadrature $(1 \& Q)$ components of the RF field. Operating upon the orthogonal $I \& Q$ components reduces the complex dynamics of amplitude and phase control to a linear control problem. The I ANL control system compensates the I\&Q components of the RF field to provide the desired steady-state and transient performance characteristics. In addi-

\footnotetext{
*Work supported and funded by the United States Department of Defense, Army Strategic Defense Command, under the auspices of the United States Department of Energy.
}

0-7803-0135-8/91503.00 @IEEE tion, the control circuitry includes computer interface circuitry that allows direct supervisory control by a microprocessor [1]. This functionality can be extended to include the microprocessor-based adaptive optimization of the various parameters in the mathematical transfer functions of the control system.

\section{SYSTEM MODEL}

The dynamics of a single-mode accelerating cavity with drive and beam stimuli are well understood [2-4]. A mathematical model exists that accurately defines the effects of external stimuli upon the RF field within a resonant cavity. This model was derived from the various intrinsic cavity parameters. Using a mathematical representation of a sinusoidal signal, the amplitude and phase information can be resolved into $I \& Q$ components as

$$
s(t)=i(t) \cos \left(\omega_{0} t\right)-q(t) \sin \left(\omega_{0} t\right) .
$$

These I\&Q components are orthogonal, creating a symmetry in the mathematical relationships of the high power amplifier and accelerating cavity, referred to as the plant. The time-domain analysis is simplified by using the complex-envelope isomorphism to eliminate the carrier frequency $\left(\omega_{0}\right)$ from the calculations [5]. This technique provides a mathematical tool to directly analyze the I\&Q components of an RF signal independently of the carrier. Consequently, the system analysis within this paper examines the baseband I\&Q components of the RF signals.

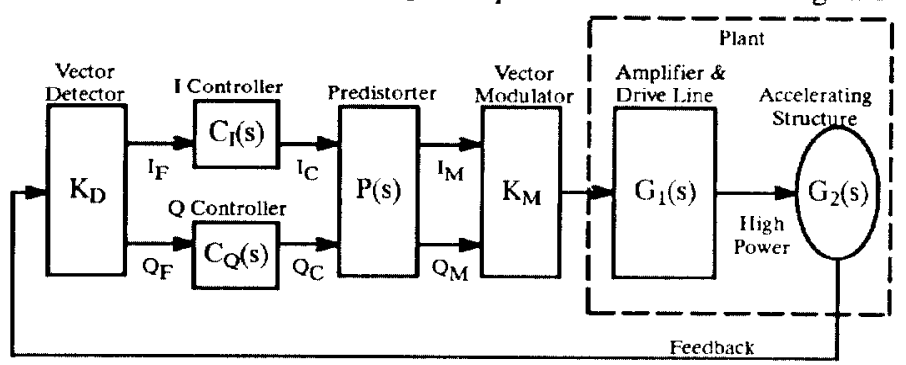

Figure 1. Simplified block diagram of I\&Q control system

To accurately maintain the RF field in the accelerating cavity, a multivariable control system regulates both I\&Q components of the field. Figure 1 depicts a simplified block diagram of the I\&Q control system. Feedback and cascade compensation is used to adjust the dynamic behavior of the plant. The I\&Q components of the cavity feedback RF signal are resolved by the vector detector into baseband analog signals that represent these quantities. Each baseband $I \& Q$ feedback signal is compared with a desired set point and the resulting error vector is compensated by the respective controller. The predistorter provides an inverse coupling function that negates any interaction between the two variables. The vector modulator uses these processed I\&Q signals to modulate the 
original RF carrier signal. The resulting modulated RF signal is subsequently used to drive the input of the plant.

The dynamics of the two-input, two-output plant in Figure 1 can be represented as a transfer function block diagram that relates the I\&Q components of the drive signal to the I\&Q components of the cavity field. Crosscoupling terms define the interactions between the two components. Coupling between the real and imaginary components of a sinusoidal signal is inherent to the dynamic behavior of the plant.

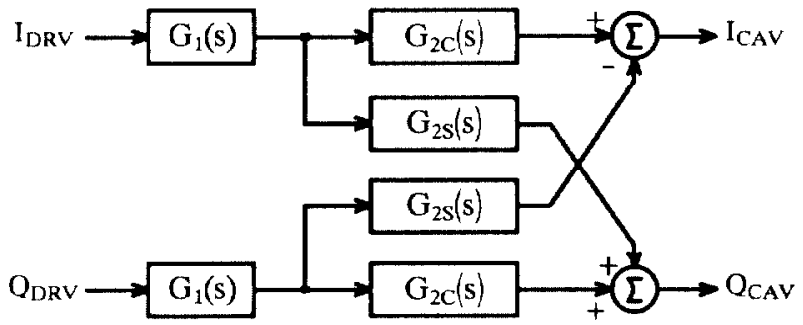

Figure 2. Diagram of cavity $I \& Q$ dynamics

Figure 2 depicts the transfer function network of a cascaded amplifier, transmission line, and accelerating cavity driven slightly off resonance. The transfer function of the cascaded RF amplifier and transmission line is

$$
G_{1}(s)=\frac{K_{A}}{\left(s / \omega_{A}+1\right)^{n}} e^{-s r_{d}} .
$$

$K_{A} \equiv \mathrm{RF}$ amplifier gain

$\omega_{A} \equiv \mathrm{RF}$ amplifier bunching cavity bandwidth

$n \equiv$ number of bunching cavities in RF amplifier

$T_{d} \equiv$ propagation delay within transmission line

Notice that the cross-coupling terms are assumed to be negligible for the RF amplifier and transmission line. The dynamics of a microwave junction and detuned cavity are described by the transfer function network terms:

$$
\begin{aligned}
G_{2 C}(s) & =\frac{2\left(Z_{C}(s)\left(Z_{C}(s)+Z_{o}\right)+Z_{S}^{2}(s)\right)}{\left(Z_{C}(s)+Z_{o}\right)^{2}+Z_{S}^{2}(s)} \quad ; \text { and } \\
G_{2 S}(s) & =\frac{2 Z_{o} Z_{S}(s)}{\left(Z_{C}(s)+Z_{o}\right)^{2}+Z_{S}^{2}(s)} \\
Z_{0} & \equiv \text { drive line characteristic impedance } \\
Z_{C}(s) & \equiv \text { cavity forward impedance function } \\
Z_{S}(s) & \equiv \text { cavity cross impedance function }
\end{aligned}
$$

The interaction between the I\&Q components within this portion of the plant dominate the cross-coupling effects within the plant. The cross-coupling terms in the plant transfer function network describe the interaction between the $1 \& Q$ components of the RF field.

\section{INVERSE COUPLING}

The cross-coupling within the plant causes interaction between the two feedback control loops that requires correction to accurately regulate the $R F$ field. To separate the $I \& Q$ dynamics, a predistorter performs an inverse coupling function. The predistorter couples the I\&Q components so that they are effectively decoupled by the plant. The function of the predistorter can be described using a transfer matrix ap- proach. To reduce the complexity of the equations, two transfer functions are defined that describe the cascaded transfer matrix for the plant of figures 1 and 2.

$$
\begin{aligned}
A(s) & \equiv K_{M} \cdot G_{1}(s) \cdot G_{2 C}(s) \\
B(s) & \equiv K_{M} \cdot G_{1}(s) \cdot G_{2 s}(s)
\end{aligned}
$$

Figure 3 shows the linear transfer matrix equation that provides a design for the predistorter. To eliminate crosscoupling within the system, the off-diagonal terms of this combined system must be identically equal to zero. In addition, the dynamics of the forward terms should remain constant regardless of the cross-coupling terms. This is represented by the factor $k_{0}$ that scales the gain of the plant to that of one without cross-coupling.

$$
\left[\begin{array}{cc}
A(s) & -B(s) \\
B(s) & A(s)
\end{array}\right]\left[\begin{array}{cc}
1 & d(s) \\
-d(s) & 1
\end{array}\right] \equiv\left[\begin{array}{cc}
k_{o} \cdot A(s) & 0 \\
0 & k_{o} \cdot A(s)
\end{array}\right]
$$

Figure 3. Transfer matrix representation of $I \& Q$ decoupling

Although the expressions for $A(s)$ and $B(s)$ are of fifth or higher order, the symmetry of the orthogonal I\&Q variables reduces the ratio of the two functions to first order. This allows the predistorter to be realized as a first order, stable, lag compensator. Solving the linear matrix equation for the two unknowns results in

$$
\begin{gathered}
d(s)=\frac{B(s)}{A(s)}=\frac{1}{2 Q_{o}}\left[\frac{s+1 / \tau+2 Q_{0} \Delta \omega}{s+1 / \tau-\Delta \omega / 2 Q_{0}+R / \tau Z_{o}}\right] \\
k_{o}=\frac{A(0)+B(0) \cdot d(0)}{A(0)} \\
=\frac{Z_{C}(0)\left(Z_{C}(0)+Z_{o}\right)+Z_{S}^{2}(0)-Z_{o} Z_{S}(0) d(0)}{Z_{C}(0)\left(Z_{C}(0)+Z_{o}\right)+Z_{S}^{2}(0)} \\
Q_{0} \equiv \text { cavity unloaded quality factor } \\
\tau \equiv \text { cavity damping time constant } \\
R \equiv \text { cavity shunt resistance } \\
\Delta \omega \equiv \text { cavity detuning frequency }
\end{gathered}
$$

A block diagram of the physical realization of the combined predistorter and plant is shown in Figure 4. This combined system will exhibit the uncoupled properties wherein an input I or Q control input will only affect the respective I or $\mathrm{Q}$ component of the cavity field. The decoupling of these two parameters has been analytically verified to better than $60 \mathrm{~dB}$ correction using a complete RF system model.

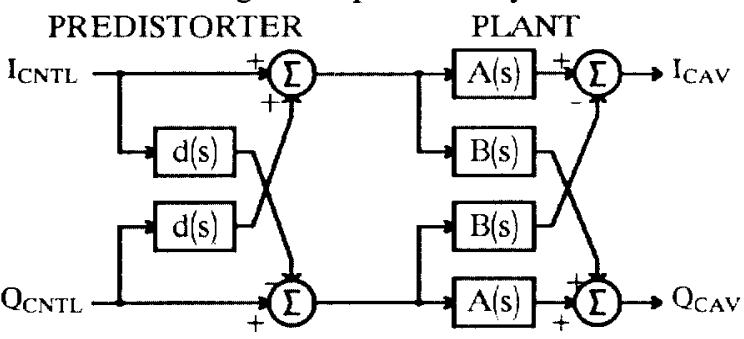

Figure 4. Block diagram representation of $I \& Q$ decoupling

The coefficients of the predistorter are adjusted remotely as the operating conditions of the plant vary. A local processor derives the coefficient values from measured sys- 
tem parameters, and automatically adjusts the hardware as necessary. In addition, an optimization algorithm can be used to correct any coefficient errors introduced by measurement uncertainties.

\section{COMPEnSATOR DESIGN}

The predistorter reduces the dynamics of the coupled system into two single-input, single-output systems that can be treated independently. Consequently, I\&Q controllers compensate the uncoupled components in parallel. Each feedback controller regulates its respective RF field component at a desired set point, rejects noise and disturbances, remains insensitive to variations in the electrical structure of the plant, and adjusts the closed-loop transient behavior.

The sensitivily of the closed-loop system to noise and disturbances or to variations in the plant parameters is inversely proportional to the loop transfer function of the cascaded compensator, plant, and feedback sensor. The loop transfer function for either of the decoupled systems can be expressed as

$$
T(s)=C(s) \cdot K_{M} \cdot G_{1}(s) \cdot G_{2 C}(s) \cdot k_{o} \cdot K_{D} .
$$

This expression provides a method to analyze the sensitivity functions versus frequency. High loop gains below the system bandwidth are desirable to provide disturbance rejection and insensitivity to parameter variations. Low loop gains are desirable at the sensor noise frequencies for immunity to these signals. The frequency responsc of the compensator is adjusted to shape the loop transfer function over frequency to meet the sensitivity criteria.

The transient response characteristics of the system can be adjusted within certain stability limitations using the same gain-shaping design techniques. The phase margin of the loop transfer function provides a method to predict the closed-loop transient behavior. The Nyquist stability criterion requires that

$$
T\left(j \omega_{C}\right)=e^{\left(180^{*}+\phi_{m}\right)}
$$

for a desired system bandwidth $\left(\omega_{c}\right)$ and phase margin $\left(\phi_{m}\right)$. This requirement specifies the exact phase of the loop transfer function at the cutoff frequency. For the accelerator RF application, the resonant cavity with a high quality factor will have the lowest frequency pole. In addition to the narrow cavity bandwidth, the plant may have nonlinear effects, such as saturation and phase droop within the RF amplifier, large propagation delays that add phase shifts, and a multitude of higher-order poles within the plant, actuators, and sensors. All of these factors affect the loop transfer function and influence the transient behavior of the system. The frequency response of the compensator is adjusted to shape the loop transfer function and create a desired transient response.

The I\&Q controllers perform the gain-shaping functionality by using phase lag-lead compensation. Within each controller, a feedback signal, providing a measurement of a detected cavity field component, is compared to a desired set point. If the feedback signal deviates from the set point, an error signal is generated. A proportional, integral, derivative (PID) control algorithm is used to process the error sig- nal and provide the required output drive signal. A phase lag compensator allows the low frequency gain to be increased (which means an improvement in steady-state behavior) without sacrificing the system bandwidth or stability margins. An integrator performs the lag compensation and essentially provides infinite gain at DC, creating zero steady-state error to a step input or step disturbance. The proportional gain stage provides instantaneous error correction for low-frequency disturbances. A phase lead compensator is used to increase the stability margin and bandwidth of the system. A differentiator performs the lead compensation to provide excess gain and phase margin at high frequencies and improve the transient behavior of the system. While the differentiator increases the high-frequency gains, its bandwidth is limited to decrease the susceptibility of the system to high-frequency noise.

The relative gains of each of the three PID terms determine the combined frequency response of the controller. This compensation allows the cascaded loop transfer function for a given plant to be shaped to provide the desired performance characteristics. In addition, the PID coefficients can be automatically adjusted by a local processor to accommodate various plant dynamic characteristics or to optimize the compensator for time-varying plant dynamics.

\section{CONClusion}

The LANL I\&Q feedback control system provides an effective method for regulating the RF field within an accelerating cavity. The flexibility that has been designed into the predistorter and $I \& Q$ controllers allows the I\&Q control sys. tem to be tailored to meet most accelerator applications. With some prior system analysis and modelling, the compensator can be adjusted to provide a desired closed-loop performance. In addition, the parameters of the control system can be empirically corrected by an autonomous optimization algorithm as the plant dynamics or operating conditions change.

\section{REFERENCES}

[1] C.D. Ziomek, "Interfacing RF control electronics to the VXIbus for the Ground Test Accelerator" Proc. NPB Technical Interchange Symposium. May, 1990.

[2] B. R. Cheo and S. P. Jachim, "Dynamic interactions between RF sources and LINAC cavities with beam loading," to be published, IEEE Trans. Elec. Dev.

[3] S.P. Jachim, "Some new methods of RF control," to be published, Proc. Linear Accelerator Conference. Septem. ber, 1990.

[4] A. Young and S.P. Jachim, "A new approach in simulating RF linacs using a general, linear real-time signal processor," to be published, Proc. Purticle Accelerator Conference. May, 1991.

[5] S. Haykin, Communication Systems. New York: John Wiley \& Sons, 1983, pp. 80-108. 